\title{
Magnetic shape memory effect progress from idea to first actuators and sensors
}

\author{
K. Ullakko, I. Aaltio ${ }^{1}$, P. Yakovenko ${ }^{2}$, A. Sozinov ${ }^{3}$, A.A. Likhachev ${ }^{2}$ and O. Heczko \\ Helsinki University of Technology, Laboratory of Biomedical Engineering, P.O. Box 2200, \\ 02015 Hut, Finland \\ ${ }_{1}$ AdaptaMat Ltd., P.O. Box 547, 02151 Espoo, Finland \\ 2 Institute for Metal Physics, Laboratory of Thermo-Mechanical Treatment, 003680 Kiev 142, \\ Ukraine \\ ${ }^{3}$ Helsinki University of Technology, Laboratory of Physical Metallurgy and Materials Science, \\ P.O. Box 6200, 02015 Hut, Finland
}

The report will review the recent progress in studies of Magnetic Shape Memory (MSM) materials and latest developments of their applications.

Magnetically controlled shape memory (MSM) materials are a new class of actuators and sensory materials. MSM materials can change their shape in a magnetic field applied due to the rearrangement of different martensite variant fractions. Inversely, magnetic field around the materials alters when proportions of the variants are changed by mechanical loading. The first effect can be utilized in actuators and the last one in sensors.

Effect of high magnetic field on the ferrous-based shape memory alloys has been observed by Kakeshita et al. (1985) and Sadovsky (1986). A new method based on redistribution of twin martensite variants by low magnetic field as well as early models of magnetic-field-induced strains were suggested by Ullakko $(1995,1996)$ and James and Wuttig (1996). Magnetic-field-induced strains of about $0.2 \%$ in non-stoichiometric NiMnGa alloy was measured by Ullakko et al. (1996). NiMnGa was selected for that research, because it is a ferromagnetic shape memory alloy and low energy is required to drive twin boundaries by stress (Martynov and Kokorin, 1992). Magnetic-fieldinduced motion of a twinning dislocation that results in a shear strain was modeled by Muellner and Ullakko (1998). MSM effect was modeled by James and Wuttig (1998), O'Handley (1998) and Likhachev and Ullakko $(1999,2000)$. Field-induced strains of $4.5 \%$ after mechanical pre-loading were measured by James et al. (1999). Shear strains of 5\% were observed by Murrey et al. (1999). The reversible elongation and contraction effect of over $5 \%$ in the rotating magnetic field was found by Ullakko, Sozinov and Yakovenko (1999).

In this report recent experimental results including reversible linear and bending strains, magnetization measurements, twin band microstructure change, fatigue and frequency response of non-stoichiometric $\mathrm{Ni}-\mathrm{Mn}-\mathrm{Ga}$ alloys as well as a new MSM model will be discussed. Presentation of functioning MSM actuators and sensors with extension-compression and also bending actuating modes will be made. 